\title{
ASTROCHEMISTRY OF COSMIC PHENOMENA: AN INTRODUCTION
}

\author{
A. DALGARNO \\ Harvard-Smithsonian Center for Astrophysics \\ 60 Garden Street \\ Cambridge, MA 02138 \\ USA
}

ABSTRACT. A general introduction is given to the subject matter of the symposium.

The first IAU Symposium on Astrochemistry took place in Goa, India in December 1985 (Tarafdar and Vardya 1987). It provided an overview of the subject matter of Astrochemistry which I take to be the formation, destruction and excitation processes of molecules in astronomical environments and the role of molecules in astronomical phenomena as diagnostic probes of the ambient physical conditions and, more fundamentally, as influences, often decisive, on the structure, dynamics and evolution of astronomical objects.

The subject has matured rapidly in the intervening years, driven like most of astronomy primarily by observational advances but helped by a developing understanding of the chemistry to the point where we can begin to use the chemical composition to draw conclusions about astronomical events, particularly those associated with star formation. We understand qualitatively that the chemical composition of the gas in regions of high and low mass star formation is modified in varying degrees by the direct effects of radiation from the stars and by the impact of material inflows and outflows accompanying the birth, evolution and death of stars. The radiation warms the grains and releases volatiles from the grain surfaces into the gas phase. The radiation also heats the gas and may change the chemistry into one in which endothermic reactions with molecular hydrogen became effective on short time scales. The outflows drive shocks which compress, heat and accelerate the gas. If rapid enough, the shocks erode and destroy the grains and dissociate and ionize the gas. The ionized gas recombines to produce a precursor radiation field that can photodissociate the molecules as they form in the cooling gas. The chemical composition of a cooling gas is quite different from that of a cold gas and quite different from that of a gas warmed by a slower non-dissociative shock.

The Orion K-L outflow region around the infrared source IRC 2 is a familiar example of a chemistry profoundly affected by massive star formation. Much higher abundances of $\mathrm{HCO}^{+}$, SO and $\mathrm{SO}_{2}$ occur in the plateau region than in the hot core. The relatively small abundance of $\mathrm{HCO}^{+}$in the hot core may reflect a low level of ionization in dense gas or it may be due to a high abundance of $\mathrm{H}_{2} \mathrm{O}$ which converts $\mathrm{HCO}^{+}$to $\mathrm{H}_{3} \mathrm{O}^{+}$by the reaction 
$\mathrm{HCO}^{+}+\mathrm{H}_{2} \mathrm{O} \rightarrow \mathrm{H}_{3} \mathrm{O}^{+}+\mathrm{CO}$. The high abundances of $\mathrm{H}_{2} \mathrm{O}$, and of $\mathrm{H}_{2} \mathrm{~S}$, may arise from their release from grain surfaces but in a warm non-dissociated gas they are produced by reactions initiated by $\mathrm{O}+\mathrm{H}_{2} \rightarrow \mathrm{OH}+\mathrm{H}$ and $\mathrm{S}+\mathrm{H}_{2} \rightarrow \mathrm{SH}+\mathrm{H}$. The reactions producing sulfur compounds are very slow in cold clouds.

The molecules $\mathrm{H}_{2} \mathrm{O}$ and $\mathrm{H}_{2} \mathrm{O}$ and $\mathrm{H}_{2} \mathrm{~S}$ are powerful coolants so that the temperature and the chemistry are intimately related.

Persuasive evidence that these regions have been modified by shock or radiation processing of grains are the observations of large abundances of deuterated species HDO, $\mathrm{NH}_{2} \mathrm{D}, \mathrm{CH}_{3} \mathrm{OD}, \mathrm{DCN}$ and $\mathrm{D}_{2} \mathrm{CO}$ in the hot core and ridge regions which suggest that the molecules were produced in a cold gas, underwent fractionation at the low temperatures, were condensed on to grains and then released less than $10^{4}$ years ago by the action of the newly formed star.

The effects of intense radiation fields on the chemical composition and cooling of interstellar gas in the absence of shocks have been explored quantitatively beginning with a study by Tielens and Hollenbach in 1985 . We know from earlier work on diffuse clouds and a model of the dense cloud L134N that in the envelopes of molecular clouds there is a transition from a gas of atomic hydrogen to a gas of molecular hydrogen in which molecular hydrogen forms and shields itself from the destructive effects of the radiation field. The regions in clouds subjected to intense radiation fields have been called photodissociation regions (PDR) by Tielens and Hollenbach and photochemical regions by van Dishoeck. Because they are regions in which molecular formation is driven by photoionization and molecular destruction is determined by photodissociation, I suggest the term "photon-dominated regions". The acronym PDR can be retained.

Photon-dominated regions occur on a larger scale in starburst galaxies and infrared emission from excited rotation-vibration levels of molecular hydrogen, produced by ultraviolet pumping by the radiation field, have been detected in external galaxies. As in objects in our galaxy, the energy source for the excitation may come also from shocks associated with star formation and supernovae. A further source of excitation is pumping by photoelectrons ejected in the absorption of X-rays. There occur differences in the emission spectra resulting from the three mechanisms and they can be distinguished by observations of transitions from high-lying levels.

Photon-dominated regions are regions in which $\mathrm{C}^{+}$is converted to $\mathrm{CO}$ and in them the $\mathrm{C}^{+} / \mathrm{O}$ and $\mathrm{C} / \mathrm{CO}$ ratios are anomalously high. If the gas density is high enough to quench vibrationally excited $\mathrm{H}_{2}$ molecules, the regions are warm and they are characterized by strong emissions of the oxygen fine-structure lines and of high rotational transitions of CO. The enhanced $\mathrm{C}^{+}$leads to infrared emission from metastable levels of neutral carbon. The emission line intensities provide a direct measure of the incident ultraviolet fluxes and the gas densities. Similar photon-dominated regions exist around hot stars and $21 \mathrm{~cm}$ radiation from the transition zones has been detected. The chemistry of PDR's has unique features that have yet to be fully explored.

Analysis of PDR's often indicates the existence of high density clumps with densities between $10^{6} \mathrm{~cm}^{-3}$ and $10^{7} \mathrm{~cm}^{-3}$. Clumpy structure may be a common phenomenon in 
PDR's and more generally in interstellar clouds.

Evidence has accumulated that even in the absence of active star formation, interstellar clouds have structure on small and large scales that may be reflected in a varying chemical composition and different spatial distributions of individual atomic and molecular species. Some part of the inferred chemical variations may be attributable to changing excitation conditions. Particularly sensitive are molecules with large dipole moments which are usually regarded as probes of high density regions. They are also the molecules most readily excited by electron impacts. Multifrequency multilevel studies are particularly valuable in separating out the possible excitation mechanisms.

On a large scale, the chemical compositions of interstellar clouds with similar densities and temperatures like TMC-1 and L134N differ, suggesting that one cloud is at an earlier stage in its evolution than the other. It may not be clear which is younger and which is older but the comparison raises again the possibility that properly interpreted the molecular composition can serve as a chemical clock. However there occur changes in composition in regions of the same clouds over scales as small as $0.2 \mathrm{pc}$. Swade has suggested the differences in $\mathrm{L} 134 \mathrm{~N}$ are due to an oxygen abundance gradient across the core of the cloud but conceivably they indicate the presence of a low-luminosity protostar that has caused some local heating. A heated gas cools more rapidly than its chemistry relaxes and a region though cold may maintain a warm chemical composition over a considerable length of time.

Such complexities make it difficult to address the important question of the distribution of a given element into its atomic, molecular and ionic forms. Gas phase oxygen that is not taken up into $\mathrm{CO}$ and $\mathrm{OH}$ is mostly in the forms $\mathrm{O}, \mathrm{H}_{2} \mathrm{O}$ and $\mathrm{O}_{2}$ and gas phase nitrogen that is not taken up into $\mathrm{NH}_{3}$ is mostly in the form $\mathrm{N}$ and $\mathrm{N}_{2}$. None of these is detectable in cold clouds. A coordinated study of different molecular species may permit some progress. For example, the abundance ratio $\mathrm{CH}_{2} \mathrm{CN} / \mathrm{CH}_{2} \mathrm{CO}$ must be related to the atomic abundance ratio $\mathrm{N} / \mathrm{O}$.

Of cosmological significance is the $\mathrm{D} / \mathrm{H}$ ratio which is a measure of the baryon density in the early Universe. Of galactic significance is the cosmic ray flux and its variations. The $\mathrm{D} / \mathrm{H}$ ratio may be inferred from observations of deuterated molecules and the cosmic ray flux from observations of molecular ions, provided that realistic models of the chemistry can be constructed.

Chemical models of a quiescent slowly varying interstellar cloud have been valuable in defining the processes that enter into interstellar chemistries but they were recognized to be no more than idealizations if only because they did not take account of the loss of gas atoms and molecules by depletion on to grains. Severely depleted regions are rare at best so that there must be mechanisms for returning the material to the gas phase or depletion must be inhibited at high densities or depleted gas has a short lifetime perhaps because it rapidly collapses.

Interstellar chemistry takes place in a complex, continuously changing, dynamically active medium in which gas forms into clouds containing clumps moving in an interclump gas and on a smaller scale dense cores moving in an intercore gas subjected to and disrupted by radiation, winds and shocks arising from star formation and during stellar evolution. The chemical response and the dynamical events are closely coupled and together control the 
continuing evolution of star-forming regions. It is one task of astrochemistry to turn this qualitative description into quantitative models and the first steps have been taken.

Chemistry may influence the clump size distributions in clouds through its control of the ionization level. Turbulence provides support against gravitational collapse. If turbulence is a superposition of Alfven waves, it will be dissipated at a rate which is sensitive to the fractional ionization. The fractional ionization varies with depth into clouds from a value of about $10^{-4}$ at the surface due to $\mathrm{C}^{+}$to about $10^{-5}$ at a visual extinction of unity due to $\mathrm{S}^{+}$followed by narrow zones of $\mathrm{Si}^{+}$and $\mathrm{Fe}^{+}$until the molecular region is reached with a fractional ionization of $10^{-7}$. Still deeper the ionization may become atomic again or the positive charge may reside on grains. The zones of diminishing ionization have different dissipation rates and different ambipolar diffusion rates. The turbulent motions may also affect the chemistry directly and perhaps be dissipated by chemical reactions.

Star formation is accompanied by a protostellar wind that collides with the remnant core material. The winds may be mostly neutral. If the density is high, the wind cools and molecular synthesis can occur. The chemistry is hydrogen rich and molecular hydrogen can be formed by reactions catalysed by electrons and protons as in the early Universe. The heart of the chemistry is the hydroxyl radical which leads to other species, particularly $\mathrm{CO}$, as in the chemistry of a dissociative shock and in photon-dominated regions.

A similar chemistry operates in the envelopes of supernovae, where $\mathrm{H}_{3}{ }^{+}$may have been detected. $\mathrm{CO}$ and $\mathrm{SiO}$ have been identified in the core of SN 1987A but there it is hydrogen poor and the major source of $\mathrm{CO}$ is the radiative association of $\mathrm{C}$ and $\mathrm{O}$ atoms.

In circumstellar shells, molecular observations are important in the determination of mass loss rates, but they also pose interesting questions of chemistry in a region where grains and photodissociation play major roles. The study of circumstellar shells may help in understanding the influence of grains in interstellar chemistry. Circumstellar chemistry has some similarities to the chemistry of cometary materials where processing by the Sun may be minimal and the connection between the solar system and the interstellar medium can be investigated. Comparing the chemical compositions, the connection is not immediately apparent and more observational data are needed to make progress.

Diffuse and translucent cloud studies and high latitude cloud studies are probably the major areas where the chemical schemes can be tested quantitatively. Problems still remain concerning the abundances of $\mathrm{CH}^{+}, \mathrm{CO}$ and $\mathrm{CN}$ that may not be resolvable by postulating shocks and a reduced ultraviolet radiation field. Perhaps dense clumps are present even in diffuse clouds. Perhaps polycyclic aromatic hydrocarbons, suggested as major repositories of carbon in the interstellar medium, participate in the chemistry.

Our galaxy provides a test bed for the effectiveness of molecules as indicators of density, temperature, radiation field intensities and cosmic ray fluxes that can then be used in the study of molecular regions of external galaxies. Many species have been detected in addition to vibrationally excited $\mathrm{H}_{2}$ and the ubiquitous $\mathrm{CO}$. Isotopic variants have been found though as yet no deuterated species.

The carbon monoxide is usually taken to be a quantitative measure of the total mass. It is important to identify other species that might test this assumption. In any event, the observations of other molecules promise to provide much insight into the nature of external 
galaxies and greatly extend the domain of astrochemistry.

The early Universe is an interesting area of astrochemistry which despite its chemical simplicity may not be fully explored. The formation of $\mathrm{H}_{2}$ was a determinant of the relict electron density after recombination and $\mathrm{H}_{2}$ played a crucial role in the initial mass spectrum of the first distinct astronomical entities. Other species may have been important in galaxy formation.

Basic data underly all the theoretical and observational advances. Here much progress has been made in quantum-chemical calculations of transition frequencies, dipole moments and excitation cross sections, in measurements at low temperatures of ion-molecule reactions, in measurements of the end-products of dissociative recombination, in measurements of the ultraviolet absorption spectrum of $\mathrm{CO}$ and the resulting calculations of the photodissociation rate of $\mathrm{CO}$ and in measurements of the rate coefficient for dissociative recombination of $\mathrm{H}_{3}{ }^{+}$which show it to be again (probably) a fast reaction. Exciting developments have taken place in interstellar and laboratory studies of polycyclic aromatic hydrocarbons and carbon clusters and in spectroscopy of molecules on surfaces. A quantitative assessment has been made of the photodissociation rates induced by cosmic rays by the Prasad-Tarafdar mechanism. Much remains to be done in all the areas of basic studies.

I hope that IAU Symposium \#150 will show that astrochemistry addresses significant astronomical issues. I expect to hear specific discussions that demonstrate the utility of astrochemistry and that identify the critical questions that need to be answered if astrochemistry is to develop further as a major branch of astronomy.

The ideas presented in this introduction were advanced by many different individuals. I have given no references. They can be found in the Invited and Contributed Papers that follow.

My research in astronomy is supported by the National Science Foundation under grant AST 89-21939 and the National Aeronautics and Space Administration under grant NAGW-1516. 


\section{QUESTIONS AND ANSWERS}

V.Burdyuzha: Why molecules with deuterium are more abundant in Ori region than in other regions of our galaxy?

A.Dalgarno: The interesting question is why they are abundant in a warm gas. Deuterated molecules are observed to be abundant in cold interstellar clouds.

D.A.Williams: Are we sometimes led astray by our terminology: e.g. what is a quiescent cloud? Such objects as TMC-1 may in fact be transient?

A.Dalgarno: I tried to use the term "apparently quiescent" because of consideration such as you present.

J.C.Pecker: Obvious "clumps" in ISM give place to some differences in the observed chemical molecular composition. Could it be due (in part?) to their fine structure, and the local unresolved physical inhomogeneities? And could the observed differences be then a somewhat spurious effect of the diagnosis difficulties?

A.Dalgarno: I think it is due to physical inhomogeneities. The question is what then is the origin of the inhomogeneities. 